\title{
Influence of shape on haptic curvature perception
}

\author{
Ingrid M.L.C. Vogels *, Astrid M.L. Kappers, Jan J. Koenderink \\ Helmholtz Instituut, Princetonplein 5, 3584 CC Utrecht, The Netherlands \\ Received 15 April 1998; received in revised form 03 August 1998; accepted 06 August 1998
}

\begin{abstract}
Curvature discrimination of hand-sized doubly curved surfaces by means of static touch was investigated. Stimuli consisted of hyperbolical, cylindrical, elliptical and spherical surfaces of various curvatures. In the first experiment subjects had to discriminate the curvature along a specified orientation (the discrimination orientation) of a doubly curved surface from a flat surface. The curvature to be discriminated was oriented either along the middle finger or across the middle finger of the right hand. Independent of the shape of the surface, thresholds were found to be about 1.6 times smaller along the middle finger than across the middle finger. Discrimination biases were found to be strongly influenced by the shape of the surface; subjects judged a curvature to be more convex when the perpendicular curvature was convex than when this curvature was concave. With the results of the second experiment it could be ruled out that the influence of shape on curvature perception was simply due to a systematic error made by the subject regarding the discrimination orientation. (C) 1999 Elsevier Science B.V. All rights reserved.
\end{abstract}

PsycINFO classification: 2320

Keywords: Haptics; Curvature perception; Shape

\section{Introduction}

Humans can use their hands to recognize objects. Lederman and Klatzky (1987) found that the strategy subjects use to explore an object depends on the nature of the

${ }^{*}$ Corresponding author. Tel.: +31 30 2532810; fax: +31 30 2522664; e-mail: a.m.1.kappers@phys.uu.nl 
information to be extracted. For instance, when the global shape of an object has to be judged subjects often use an exploratory procedure called 'contour following'. The strategy subjects naturally use is not always the optimal one. When subjects are restricted to a prespecified exploratory procedure, 'static contact' results in the most accurate judgements of global shape. Apparently, useful information about the shape of an object can be extracted by only resting one hand passively on its surface. Pont et al. (1997) demonstrated that also the curvature of an object can be extracted by static touch. ${ }^{1}$ They measured thresholds for static curvature discrimination of $20 \mathrm{~cm}$ long strips (or 'singly curved surfaces') touched with different parts of the hand. Discrimination thresholds for strips placed across the fingers were twice as high as for strips presented along the fingers. The length of the contact area of the hand with the strips appeared to be a determining factor for performance, not the anatomical or neural structure of the part of the hand that touched the strip. This result is in agreement with their finding (Pont et al., 1998a) that the slope difference over the strip represents the effective stimulus for curvature perception. When stimulus length is increased, slope differences increase and hence discrimination thresholds decrease. Moreover, longer strips will be judged to be more curved than shorter strips with the same physical curvature. This result was observed for several conditions: when the two strips to be discriminated were touched with the same part of the hand (Pont et al., 1998a), when the index fingertip moved along the surface of the strips (Pont et al., 1998a) and when one strip was touched along the index finger and the other across the fingers (Pont et al., 1998b).

The increase in perceived curvature with increasing contact length can be extrapolated to hand-sized surfaces. Pont et al. (1998b) showed that the curvature of cylinders touched along the fingers was overestimated in comparison with the curvature of cylinders touched across the fingers. Moreover, they found that curvature comparison was biased, i.e. the perceived (phenomenal) flatness along the fingers differed from that across the fingers. In a shape discrimination experiment they found that the perceived shape of a doubly curved surface was influenced by its orientation. ${ }^{2}$ This orientation effect could be predicted from the influence of contact length on the perceived curvature of a cylinder and the difference in phenomenal flatness along and across fingers.

In this paper we extend the study of static curvature perception of hand-sized stimuli which are touched with the entire hand. Firstly, we investigate whether the influence of contact length on thresholds of curvature discrimination can be extrapolated to hand-sized surfaces. Since Pont et al. (1998b) found that the perceived curvature of a cylinder depends on contact length, we expect to find the same effect for thresholds of curvature discrimination. Secondly, we examine whether thresholds depend on the shape of the surface. It might conceivably be easier for subjects to

\footnotetext{
${ }^{1}$ Because in their experiments static touch had to be preceded by a movement of the hand relative to the object, strictly speaking this procedure should be called 'active static touch'.

${ }^{2}$ The shape of a smooth three-dimensional object is determined by the distribution of curvatures on its surface.
} 
distinguish a curvature from flat when the curvatures along all other orientations have the same sign (an elliptical surface) than when they have opposite signs (a hyperbolical surface). Thirdly, we investigate whether the discrimination bias, which corresponds to the difference in phenomenal flatness along a particular orientation of the two surfaces (a doubly curved and a flat surface) to be discriminated, depends on the shape of the doubly curved surface. Since Pont et al. (1998b) found that for cylinders the phenomenal flatness differs along and across the fingers, it is interesting to study the phenomenal flatness for other curved surfaces as well.

Besides the studies of Pont et al. (1998a,b, 1997) the following studies are of relevance for our research. Hunter (1954) and Davidson (1972) showed that if subjects actively explored an edge of $20 \mathrm{~cm}$ length with the fingertips, they judged the edge to be straight when it was curved away from the subject. The scanning technique appeared to affect the veridicality and accuracy of the judgements. Goodnow et al. (1971) showed that symmetrically curved edges are often felt to be skewed, the direction of the skew depending on the direction of the scanning movement. Gordon and Morison (1982) tested dynamic curvature discrimination of curved strips $(10 \times$ $20 \mathrm{~mm}^{2}$ ) with the index finger. They found that a strip with a curvature of $2 \mathrm{~m}^{-1}$ was discriminable from a flat surface. ${ }^{3}$ Goodwin et al. (1991) found that a flat surface applied to the fingerpad could be distinguished from a convex surface with a curvature of $+4.9 \mathrm{~m}^{-1}$ and from a concave surface with a curvature of $-5.4 \mathrm{~m}^{-1}$. Kappers and Koenderink (1996) showed that dynamic curvature discrimination of hand-sized cylindrical surfaces did not follow a Weber law; performance was relatively better with larger curvatures. In the study of Vogels et al. (1996) subjects had to judge the curvature of a hand-sized spherical surface by means of static touch, after they had touched a conditioning surface. It was found that the curvature of the conditioning surface had an influence on which surface was judged to be flat, but not on the threshold of curvature detection, which was about $0.25 \mathrm{~m}^{-1}$.

LaMotte and Srinivasan performed neurophysiological experiments to investigate how shape is represented in the activity of cutaneous mechanoreceptive nerve fibers (LaMotte and Srinivasan, 1993; LaMotte et al., 1994; Srinivasan and LaMotte, 1987). They recorded the responses of single peripheral nerve fibers to different stimuli indented into and stroked across the primate fingerpad. Their stimuli consisted of sinusoidally shaped steps, cylindrical bars, ellipsoids and spheres. They showed that the geometrical features of the shape of an object are represented in the spatiotemporal responses of slowly adapting (SA) and rapidly adapting (RA) cutaneous mechanoreceptors. Intensive information, such as the magnitude of change in skin curvature due to contact with the object, is provided in the discharge rates of SAs and RAs. Spatial information, such as the size and shape of an object, is represented best in the shape of the spatial distribution of discharge rates in SAs. This spatial response profile provides a neural code that is probably invariant with moderate changes in the way the object contacts the skin.

\footnotetext{
${ }^{3}$ Curvature is defined as reciprocal radius. So, a sphere with a radius of $0.5 \mathrm{~m}$ has a curvature of $2 \mathrm{~m}^{-1}$.
} 
When small stimuli are applied to the fingerpad cutaneous mechanoreceptors are essential for curvature and shape perception. As mentioned, the threshold for curvature discrimination is then about $5 \mathrm{~m}^{-1}$ (Goodwin et al., 1991). When hand-sized surfaces are touched statically with several fingers or the entire hand thresholds are an order of magnitude lower (Pont et al., 1997; Vogels et al., 1996). Judgements of curvatures less than $5 \mathrm{~m}^{-1}$ cannot be curvatures at the skin. Kinesthetic information about the position of finger joints, which is primarily provided by muscle spindle receptors (Matthews, 1988), could play a role. Pont et al. (1998a) reported that curvature perception of curved strips ranging from $-4 \mathrm{~m}^{-1}$ to $4 \mathrm{~m}^{-1}$ is based on local attitude differences. The kinesthetic sense does not contribute directly to curvature perception but it could be involved in determining the positions of the fingers relative to each other.

In this paper we used doubly curved surfaces (see Eq. 1) to investigate static curvature perception for hand-sized surfaces. For these surfaces the curvature varies with the orientation along the surface. The two orthogonal axes along which the curvature has an extreme value are called the principal axes. The aim of the first experiment was to investigate whether subjects are able to judge the curvature of a doubly curved surface along a specified orientation. Determining the shape of a surface can be done by judging the curvature along particular orientations and therefore it is interesting to study how well subjects can do this. Subjects were asked to discriminate the curvature along one of the two principal axes of a doubly curved surface with the curvature of a flat surface. (We used a discrimination task to avoid variations due to after-effects, see Vogels et al., 1996). Since the curvatures along all other orientations are different this could complicate or disturb the judgement along the specified orientation. Therefore we investigated the influence of shape on the bias and threshold of curvature discrimination by varying one of the principal curvatures while the principal curvature to be discriminated was kept constant. We also varied the orientation of the curvature to be discriminated in order to investigate whether discrimination thresholds depend on the orientation of the curvature relative to the hand. Pont et al. (1997) demonstrated that discrimination performance for curved strips is better for strips placed along the fingers than for strips across the fingers. It could be that this is the same when the entire hand is stimulated instead of only a small part of the hand.

In experiment 2 we tested whether subjects were actually able to pay attention to the right orientation and if not whether that could explain the influence of shape on curvature perception found in experiment 1 .

\section{Experiment 1}

The aim of this experiment was two-fold. First, we investigated the ability of human observers to distinguish the curvature of a doubly curved surface from a flat surface, both along the middle finger and across the middle finger. The ease with which one can curve the hand and the range of possible curvatures are very different for the fingers and the palm of the hand. In spite of this, Pont et al. (1997) 
demonstrated that for curved strips the performance of curvature discrimination is independent of the part of the hand that makes contact with the strip, i.e. the morphology of the hand is not important. Performance was found to depend on the length of the contact area of the hand with the strip. Since the contact length along the fingers is larger than across the fingers, performance is better for strips placed along the fingers. In our experiment the length of the contact area is not defined, since the entire hand has to make contact with the surface. However, thresholds could depend on the length of the contact area along the orientation of the curvature that has to be discriminated. Performance would then be better for curvature discrimination along the middle finger than across the middle finger. If performance depends on contact area, thresholds should be the same for the two orientations, since the contact area is always the same.

Our second question was whether the shape of the surface affected the threshold and bias of curvature discrimination. In order to judge the curvature along a specified orientation one has to integrate information over a particular part of the hand and ignore information from the rest of the hand. The information is coming from many different muscle, joint and cutaneous receptors. It is not a priori obvious whether the central nervous system can select the right information and integrate it correctly to obtain a curvature percept. It is, however, conceivable that the system cannot completely ignore irrelevant information. Therefore, it could be that the presence of different curvatures with different orientations complicates or disturbs curvature discrimination along a specified orientation.

\subsection{Method}

\subsubsection{Stimuli}

In all our experiments we used doubly curved surfaces, which can be described mathematically as:

$$
z(x, y)=-\frac{1}{2}\left(k_{\min } x^{2}+k_{\max } y^{2}\right)
$$

where the coordinates $(x, y, z)$ are measured along the axes of an orthonormal frame. The coefficients $k_{\min }$ and $k_{\max }$ are the two principal curvatures. A positive (negative) curvature will be called convex (concave). By varying $k_{\min }$ and $k_{\max }$ one can obtain all kinds of convex and concave, elliptical and hyperbolical paraboloids of different curvatures. Because at any point of a smooth surface one can approximate the surface in a sufficiently small neighbourhood as a doubly curved surface, Eq. 1 describes all possible local surfaces up to the second order.

For doubly curved surfaces the normal curvature $k$ of a path cut out by a normal plane through a point on the surface depends on the orientation of the normal plane: ${ }^{4}$

\footnotetext{
${ }^{4}$ Because the size and maximum curvature of our surfaces are sufficiently small the variation in the curvature along that path can be neglected.
} 


$$
k(\varphi)=k_{\max } \cos ^{2}(\varphi)+k_{\min } \sin ^{2}(\varphi) \Rightarrow k_{\min } \leqslant k(\varphi) \leqslant k_{\max },
$$

where $\varphi$ is the angle between the orientation of the normal plane and the orientation of $k_{\max }$. The shape of doubly curved surfaces is the same at each point of the surface; it only depends on the values of $k_{\max }$ and $k_{\min }$.

\subsubsection{Experimental setup}

The stimuli were made from polyurethane foam impregnated with synthetic resin. The mechanical structure of this composite is comparable to beechwood. The stimuli had a doubly curved upper surface, as described by Eq. 1, and a flat bottom, which rested on a table. The diameter of all stimuli was $20 \mathrm{~cm}$ and the height at the middle of the stimuli was $4.5 \mathrm{~cm}$. In Fig. 1 a number of different doubly curved surfaces are schematically illustrated. For clarity, the curvatures of the drawn surfaces are much larger than the curvatures of the surfaces used in this experiment. The principal curvatures $k_{\max }$ and $k_{\min }$ could have a value of $-1,-0.7,-0.5,-0.35,0,0.35,0.5,0.7$ or $1 \mathrm{~m}^{-1}$. The height difference between the middle of the stimulus and the edges was at most $5 \mathrm{~mm}$, which indicates that the curvatures are rather small and the surfaces can easily be touched with the entire hand. All combinations of $k_{\max }$ and $k_{\min }$ were possible, except those for which one of the principal curvatures was -0.7 or $0.7 \mathrm{~m}^{-1}$ and the other -1 or $1 \mathrm{~m}^{-1}$. These combinations were omitted in order to restrict the duration of the experiment. Since $k_{\max } \geqslant k_{\min }$, we had 41 distinct shapes.

\subsubsection{Subjects}

Seven naïve paid subjects participated in this experiment. Subjects MK, WR, BH, RN and GO were strongly right-handed, subject RR was weakly right-handed and subject SW was strongly left-handed. The degree of left/right-handedness is defined by Coren (1993). None of the subjects reported any haptic deficiencies.

\subsubsection{Procedure}

Subjects were seated behind a curtain at a table. They put their right hand under the curtain in order to touch the stimuli without seeing them. Their elbow rested on the table and the forearm was fixated in such a way that subjects only could move their arm up-downwards. All surfaces were placed at the same position with respect to the thorax. Subjects were instructed to put their entire hand (palm and fingers) on a stimulus such that the time of contact with the surface was about the same for all
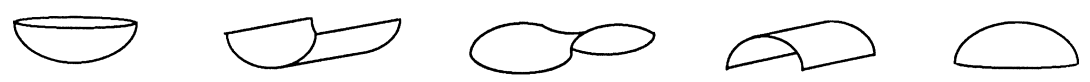

concave sphere concave cylinder saddle convex cylinder convex sphere

Fig. 1. A few examples of doubly curved surfaces. The shape of a doubly curved surface can be hyperbolical, cylindrical, elliptical or spherical. For the spherical surfaces $k_{\max }$ and $k_{\min }$ are equal, for the cylindrical surfaces either $k_{\max }$ or $k_{\min }$ is zero and oriented along the axis of the cylinder, and, for the saddle surface $k_{\max }=-k_{\min }$. The axes of the two principal curvatures are always perpendicular. 
parts of the hand, i.e. they were not allowed to roll their fingers over the surface. The fingers were not kept together, nor spread out maximally, but they were in a comfortable position. Once the hand had touched the surface, subjects were not allowed to move their hand. Because subjects could not make left-right movements with their forearm the position of the hand on the surface was very stereotype. To be sure that the orientation of the hand relative to the surface was correct, the experimentor checked whether the middle finger was aligned with a line drawn on the surface. If not, the trial was repeated without knowledge of the subject.

In each trial two surfaces, a flat reference surface and a doubly curved one, were presented successively to the subject. The order of each stimulus pair was randomized so that the subject did not know which of the two stimuli was the flat one. The task of the subject was to pay attention to the curvature along a specified orientation, called the 'discrimination orientation'. Subjects were instructed to touch the first surface and remove their hand as soon as they were confident about the curvature to be judged. Then they touched the secondly presented surface and judged whether this surface was more convex or more concave along the discrimination orientation. Subjects touched each of the two surfaces only once. They were never given any feedback.

As shown in Fig. 2, the orientation to which they had to attend, indicated by an arrow, could either be the orientation of the middle finger $\left(0^{\circ}\right.$ condition $)$ or perpendicular to (i.e. across) the middle finger $\left(90^{\circ}\right.$ condition). The surfaces could be presented in two orientations relative to the hand: $0^{\circ}$ and $90^{\circ}$. The orientation was defined as the angle between the middle finger and the principal axis of $k_{\max }$. So, subjects had to discriminate either the curvature of $k_{\max }$ or $k_{\min }$. The curvature to be discriminated will be called $k_{\mathrm{d}}$, and the perpendicular principal curvature will be called $k_{\mathrm{p}}$. Two surfaces with the same shape but different orientations were considered as different stimuli, because the curvatures to be discriminated were different. Hence, we had $73(32 \times 2+9)$ stimuli in this experiment (the nine spherical surfaces have the same curvature along each orientation). For subjects BH, RN, SW and GO we only presented those stimuli for which $k_{\mathrm{p}}$ was $-1,-0.5,0,0.5$ or $1 \mathrm{~m}^{-1}$. For subjects MK, WR and RR we also presented stimuli for which $k_{\mathrm{p}}$ was $-0.7,-0.35$, 0.35 or $0.7 \mathrm{~m}^{-1}$. Each stimulus was presented 10 times for each of the two discrimination orientations. The total number of presentations was thus 820 (41 stimuli $\times 2$ orientations $\times 10$ presentations) for subjects $\mathrm{BH}, \mathrm{RN}, \mathrm{SW}$ and GO and 1460 $(73 \times 2 \times 10)$ for subjects MK, WR and RR. All presentations were randomly distributed over 6 or 10 sessions, but in such a way that the orientation to which the subject had to attend was constant during one session. The sessions were measured on different days and took about three quarters of an hour each. The experiment involved about $40 \mathrm{~h}$ in total.

\subsection{Results}

In Fig. 3(a) we show the results of an ideal observer giving veridical answers. The percentages of judgements in which the doubly curved surfaces were judged to be more convex than the flat one, either along the middle finger or across the middle 


\section{discrimination orientation}

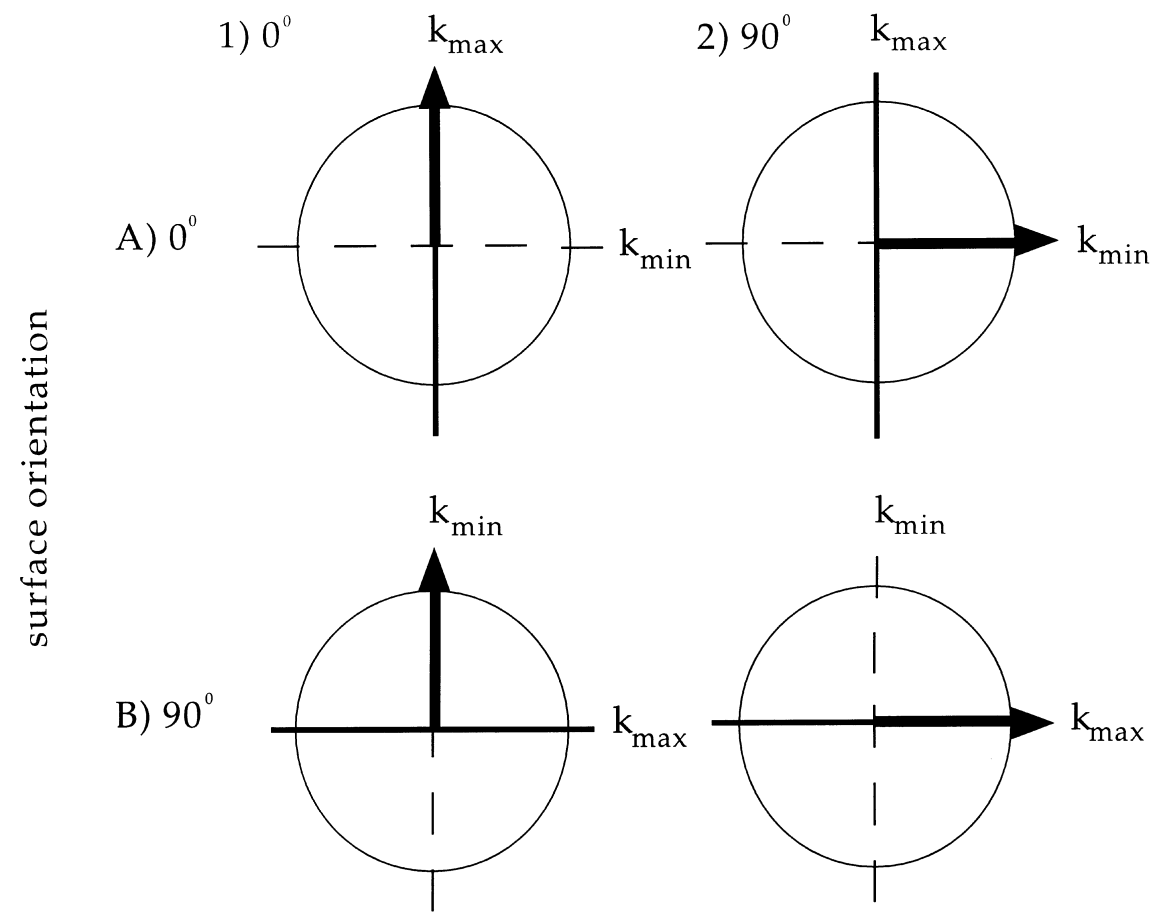

Fig. 2. The four different situations in which each doubly curved surface with principal curvatures $k_{\max }$ and $k_{\min }$ could be presented. The orientation of the surface, which corresponds to the orientation of $k_{\max }$, could be $0^{\circ}$ (a) or $90^{\circ}$ (b). Each surface orientation is considered as a different stimulus. The discrimination orientation could be $0^{\circ}(1)$ or $90^{\circ}(2)$. Each discrimination orientation is considered as a different condition. We define the orientation of the middle finger as $0^{\circ}$. The thick arrow points along the discrimination orientation, the solid line is the principal axis of $k_{\max }$, and the dashed line corresponds to the principal axis of $k_{\min }$.

finger, are presented as gray values. The vertical axis corresponds to the principal curvature that had to be discriminated $\left(k_{\mathrm{d}}\right)$, the horizontal axis represents the other perpendicular principal curvature $\left(k_{\mathrm{p}}\right)$. When the curvature to be discriminated is convex, flat or concave the percentage of judgements "more convex" of an ideal observer would be 100, 50 and 0, respectively. In Fig. 3(b) we show for each combination of $k_{\mathrm{d}}$ and $k_{\mathrm{p}}$ the corresponding shape of the surface. When the signs of the two principal curvatures are the same the surface is elliptical, when the signs are opposite the surface is hyperbolical, and, when one of the principal curvatures is zero the surface is cylindrical. It can be seen that the judgements of this ideal observer are independent of the shape of the surface.

In Fig. 4 the judgements of subject RR are presented for both the $0^{\circ}$ condition (a) and the $90^{\circ}$ condition (b), in the same way as in Fig. 3. Results of the other subjects 

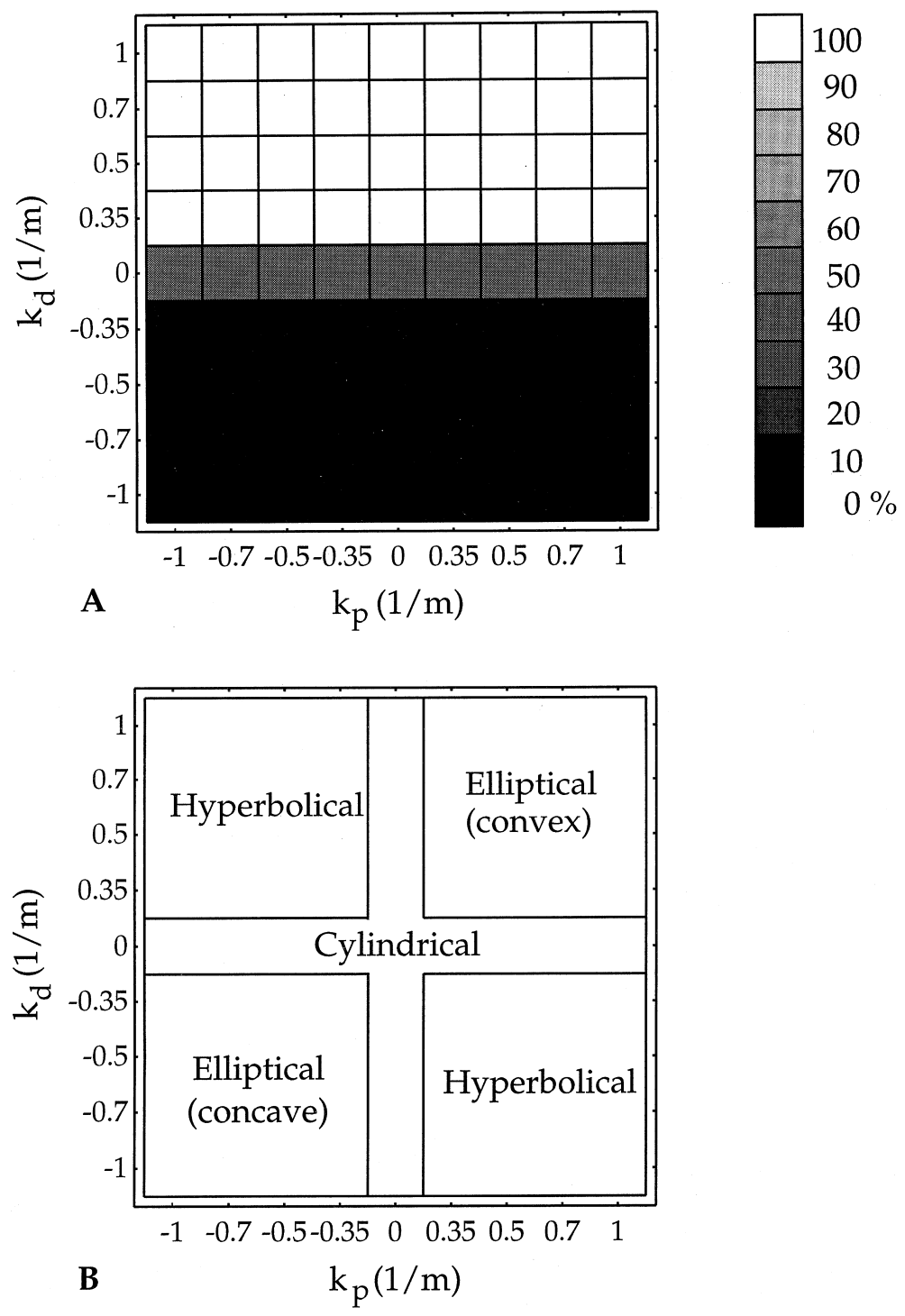

Fig. 3. (a) The percentage of judgements in which an ideal observer would judge the doubly curved surfaces to be more convex than the flat reference surface along the middle finger or across the middle finger. The percentages are plotted as gray values, according to the scale next to the diagram. Each square in the diagram represents a surface with principal curvatures $k_{\mathrm{d}}$ and $k_{\mathrm{p}}$. The curvature $k_{\mathrm{d}}$ had to be discriminated, so the judgements are independent of $k_{\mathrm{p}}$. (b) The corresponding shape of the surfaces.

will be presented later in this paper in a slightly modified way. A striking result for subject RR is that the judgement of $k_{\mathrm{d}}$ is not the same for different values of $k_{\mathrm{p}}$, in the $0^{\circ}$ condition. In other words, judgements are dependent of the shape of the surface. In the $90^{\circ}$ condition the results look more like those of the ideal observer. 

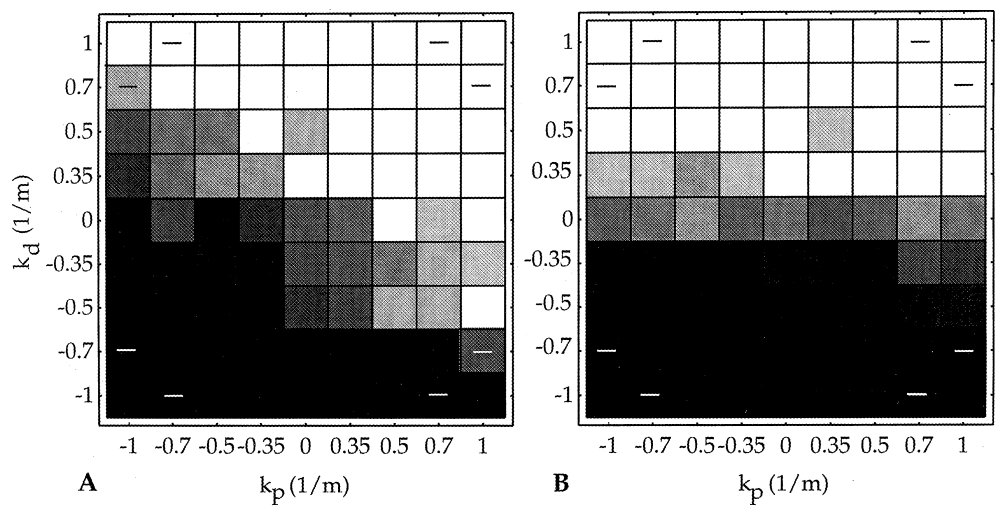

Fig. 4. The percentage of judgements "more convex" for subject RR in the $0^{\circ}$ condition where the subject had to attend to the orientation of the middle finger (a) or the $90^{\circ}$ condition where the subject had to attend to the orientation perpendicular to the middle finger (b). The scale of the gray values is the same as in Fig. 3. The squares with a minus sign represent surfaces that were not presented. In order to prevent these 'empty' squares blurring a possible trend, we gave these squares a gray value equal to the average of the three neighbouring squares.

In order to quantify the data we fitted psychometric functions (error functions or 'erf'), according to the Levenberg-Marquardt method (Press et al., 1988), for each set of stimuli that had the same value of $k_{\mathrm{p}}$ and for each condition separately. We fitted the function:

$$
p\left(k_{\mathrm{d}}\right)=50\left(1+\operatorname{erf}\left[\left(k_{\mathrm{d}}-\mu\right) / \sqrt{2} \sigma\right]\right),
$$

$\mu$ is the bias and $\sigma$ the threshold. The bias corresponds to the $50 \%$ point of the psychometric curve and represents the curvature of $k_{\mathrm{d}}$ that is judged to be the same as the curvature of the flat reference surface. The threshold is the difference between the $50 \%$ and $85 \%$ points and is inversely related to the steepness of the curve. In Fig. 5 an example of a psychometric function is shown for $k_{\mathrm{p}}=+0.5 \mathrm{~m}^{-1}$ and the $0^{\circ}$ condition, for subject RR. In the case of the ideal observer the curve would be a step function.

For some subjects and some values of $k_{\mathrm{p}}$ the bias of the psychometric curve was so large that the percentage 'more convex' did not vary from 0 to 100 for the presented range. Therefore, the values of the bias and threshold obtained by fitting were not reliable. This situation only occured in 5 out of 94 cases. We omitted these values in the following analysis.

We performed a multi-way ANOVA to test whether the discrimination threshold depended on the principal curvature $k_{\mathrm{p}}$, the discrimination orientation or the subject which performed the task. All measured thresholds were included in the analysis. The effect of discrimination orientation was highly significant $\left(F_{1,73}=14.2, p<\right.$ $0.001)$. The effect of principal curvature $k_{\mathrm{p}}\left(F_{8,73}=1.5, p>0.05\right)$ and subject $\left(F_{6,73}=1.9, p>0.05\right)$ failed to achieve statistical significance. There were no significant interactions. The threshold averaged over the subjects and the different values 


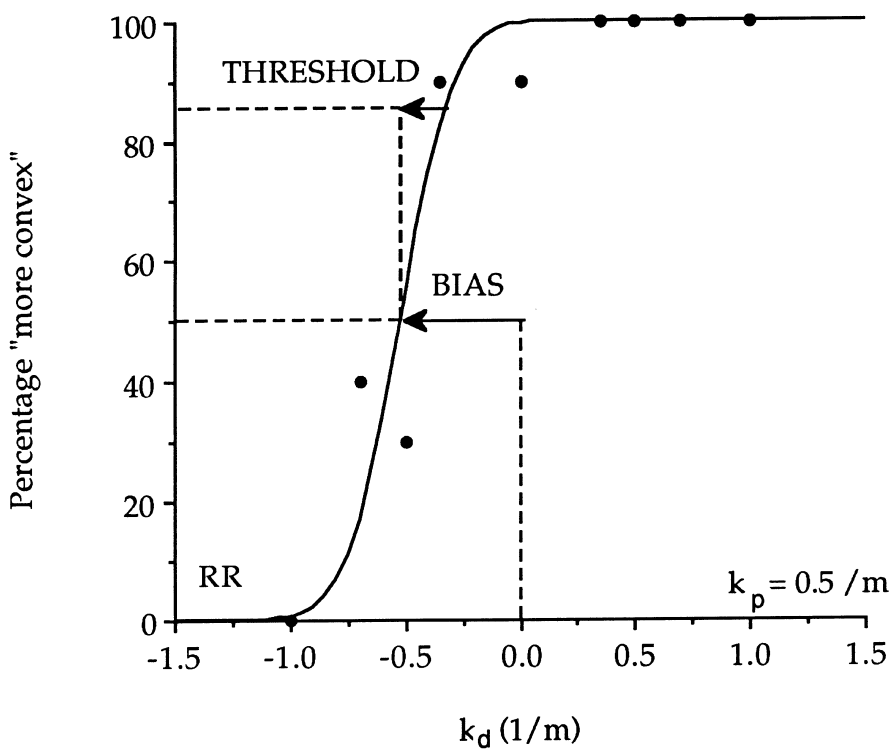

Fig. 5. The percentage of judgements "more convex" as a function of the curvature in the discrimination orientation, for $k_{\mathrm{p}}=0.5 \mathrm{~m}^{-1}$, the $0^{\circ}$ condition and subject RR. A psychometric function was fitted to the data points, according to Eq. 3 . The bias was $-0.52 \mathrm{~m}^{-1}$, the discrimination threshold was $0.19 \mathrm{~m}^{-1}$.

of $k_{\mathrm{p}}$ was $0.20 \mathrm{~m}^{-1}$ for a discrimination orientation of $0^{\circ}$ and $0.32 \mathrm{~m}^{-1}$ for a discrimination orientation of $90^{\circ}$.

In Fig. 6 we plotted the bias of the psychometric curves as a function of $k_{\mathrm{p}}$, for all subjects. The bias varied with $k_{\mathrm{p}}$, which is in agreement with the observation of Fig. 4. The relation between the bias and $k_{\mathrm{p}}$ can be approximated well by a linear function: $\mu=a_{0}+a_{1} k_{\mathrm{p}}$. We used a regression analysis to determine the values of $a_{0}$ and $a_{1}$ and to test whether they were significantly different from zero. The offset $a_{0}$ corresponds to the curvature of $k_{\mathrm{d}}$ that is judged to be the same as the curvature of the flat surface in the case where $k_{\mathrm{p}}$ is $0 \mathrm{~m}^{-1}$. Only in one case was $a_{0}$ significantly different from $0 \mathrm{~m}^{-1}(p<0.05)$, namely for subject $\mathrm{GO}$ and the $90^{\circ}$ condition. However, the offset was negligible compared to the value of the slope $a_{1}$. The values of the slope and their standard deviations are shown in Table 1 . In the $0^{\circ}$ condition $a_{1}$ was significantly different from zero for all subjects, except subject GO. In the $90^{\circ}$ condition this was true for all subjects, except subjects WR and RR. In order to determine whether the discrimination orientation significantly influenced the observed bias we performed a multiple regression analysis with $k_{\mathrm{p}}$, the discrimination orientation and their interaction as variables. The effect of $k_{\mathrm{p}}$ was of course significant, but also the interaction between $k_{\mathrm{p}}$ and the discrimination orientation was significant for all subjects, except one. The $t$-value of the interaction was: $t_{14}=2.5, p<0.05(\mathrm{MK}) ; t_{14}=2.3, p<0.05(\mathrm{WR}) ; t_{14}=11.4$, $p<0.001 \quad(\mathrm{RR}) ; t_{6}=2.4, p>0.05 \quad(\mathrm{BH}) ; t_{5}=5.1, \quad p<0.01 \quad(\mathrm{RN}) ; t_{4}=9.3$, $p<0.001(\mathrm{SW}) ; t_{4}=5.1, p<0.01(\mathrm{GO})$. The interaction can be interpreted as an 

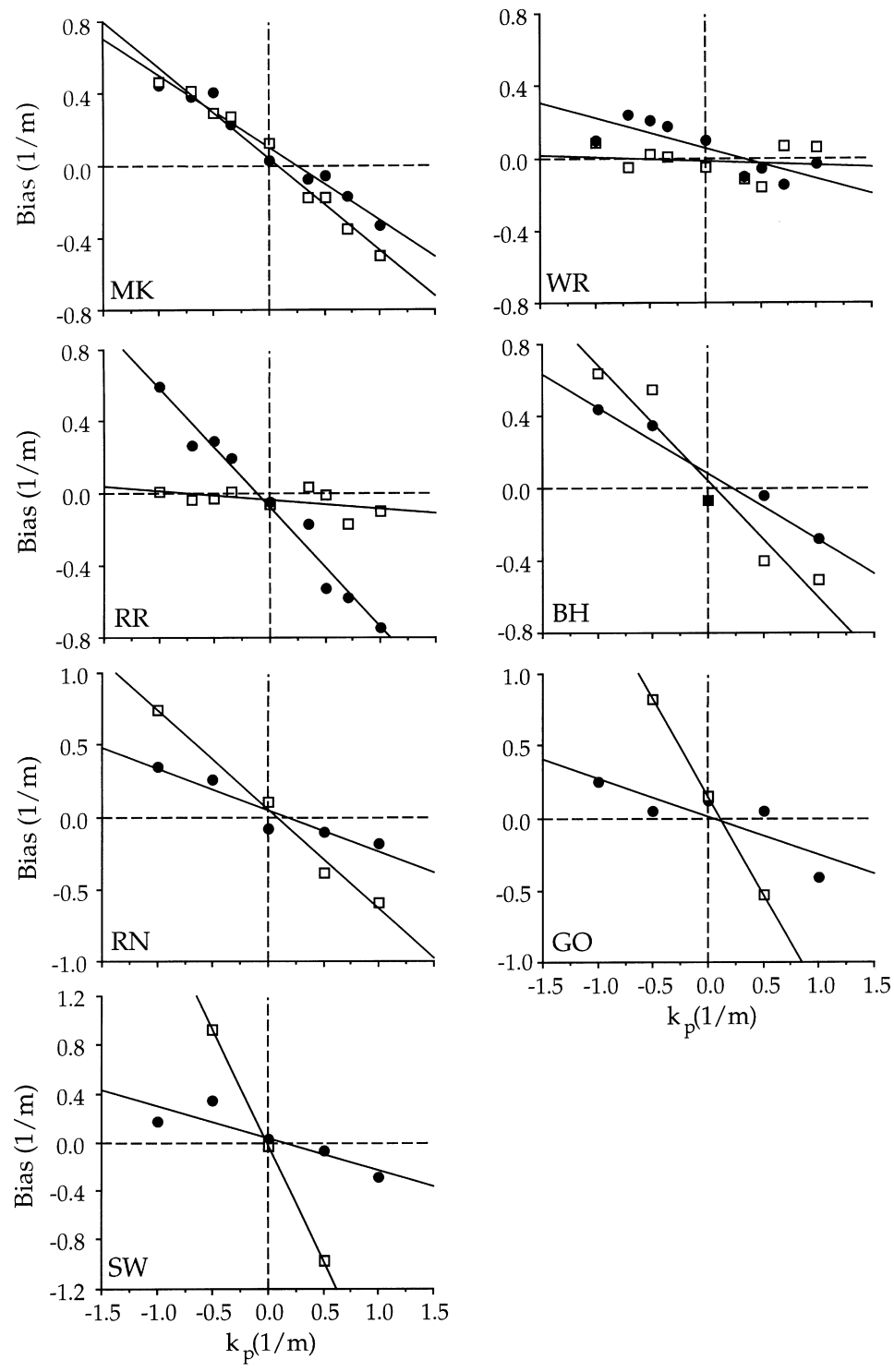

Fig. 6. The bias $(\mu)$ of the discriminated principal curvature as a function of the perpendicular curvature $k_{\mathrm{p}}$, for all subjects and both discrimination orientations: $0^{\circ}$ (filled circles) and $90^{\circ}$ (open squares). A linear function was fitted to the data points: $\mu=a_{0}+a_{1} k_{\mathrm{p}}$. The offset $a_{0}$ was never significantly different from $0 \mathrm{~m}^{-1}$, the values of the slope $a_{1}$ are presented in Table 1 .

influence of the discrimination orientation on the bias depending on the value of $k_{\mathrm{p}}$. In other words, the slopes of the lines in Fig. 6 depend significantly on the discrimination orientation. 
Table 1

Slopes and standard deviations of the slopes (SD) of straight lines fitted to the data points of Fig. 6. Slopes significantly different from 0 are marked with ${ }^{*}(p<0.05)$ or ${ }^{* *}(p<0.01)$

\begin{tabular}{llllll}
\hline Subject & $0^{\circ}$ & & & $90^{\circ}$ & \\
\cline { 2 - 3 } \cline { 5 - 6 } & Slope & SD & & Slope & SD \\
\hline MK & $-0.40^{* *}$ & 0.03 & & $-0.51^{* *}$ & 0.03 \\
WR & $-0.17^{* *}$ & 0.05 & & -0.02 & 0.05 \\
RR & $-0.66^{* *}$ & 0.04 & & -0.05 & 0.03 \\
BH & $-0.37^{*}$ & 0.10 & & $-0.65^{* *}$ & 0.07 \\
RN & $-0.28^{*}$ & 0.05 & & $-0.69^{* *}$ & 0.06 \\
SW & $-0.27^{*}$ & 0.08 & & $-1.90^{* *}$ & 0.01 \\
GO & -0.26 & 0.10 & & $-1.34^{* *}$ & 0.05 \\
\hline
\end{tabular}

\subsection{Discussion}

The discrimination of a principal curvature aligned with the middle finger or across the middle finger has been investigated. Discrimination thresholds were found to be independent of the shape of the surface, i.e. $k_{\mathrm{p}}$. Thresholds depended significantly on the orientation of the curvature to be discriminated. Averaged over all subjects, performance along the middle finger was 1.6 times better than across the middle finger. This result is in agreement with the finding of Pont et al. (1997) for curved strips. They showed that the difference in performance was mainly the result of different contact lengths of the hand with the stimulus. In the present experiment we can speak only of contact area. Since the contact area of the hand was always the same, it cannot explain the difference in performance between the two discrimination orientations. However, if the length of the contact area along the discrimination orientation is of decisive importance, thresholds could be described in terms of 'effective contact length'.

Discrimination thresholds for doubly curved surfaces $\left(0.20-0.32 \mathrm{~m}^{-1}\right)$ are smaller than for curved strips $\left(0.5-0.9 \mathrm{~m}^{-1}\right.$; Pont et al., 1997) but comparable to thresholds for curvature detection for spherical surfaces $\left(0.25 \mathrm{~m}^{-1}\right.$; Vogels et al., 1996). This indicates that subjects combined information from several parts of the hand in order to judge the curvature along a particular orientation.

Discrimination biases depended on the shape of the surface, since they varied linearly with the principal curvature perpendicular to the discriminated one $\left(k_{\mathrm{p}}\right)$. A principal curvature was judged to be more convex when $k_{\mathrm{p}}$ was convex than when $k_{\mathrm{p}}$ was concave. The influence of $k_{\mathrm{p}}$ was significant in eleven of the fourteen cases. The bias was on average as large as $-0.5 \mathrm{~m}^{-1}$ when $k_{\mathrm{p}}$ was $1 \mathrm{~m}^{-1}$. Except for two subjects, the overall trend indicated that the bias was larger when the discrimination orientation was oriented across the middle finger than when it was oriented along the middle finger. At the moment we cannot explain why the results are different for the two subjects. Possibly differences in the ratio of length and width of the subjects' hands play a role. Pont et al. (1998b) also found that for cylindrical surfaces the difference between the phenomenal flatness along and across the fingers was subjectdependent. 
In this experiment we assumed that subjects were able to attend to the specified orientation. However, if they made a systematic error in the discrimination orientation $\left(\Delta \varphi_{\mathrm{s}}\right)$ the perception of curvature along the specified orientation would be biased. The curvature discriminated by the subjects would not be the principal curvature $k_{\mathrm{d}}$, but a combination of $k_{\mathrm{d}}$ and $k_{\mathrm{p}}$, namely: $k_{\mathrm{d}} \cos ^{2}\left(\Delta \varphi_{\mathrm{s}}\right)+k_{\mathrm{p}} \sin ^{2}\left(\Delta \varphi_{\mathrm{s}}\right)$. Curvature discrimination would also be biased if subjects averaged the curvature over an area of which the boundary lines make an angle of $-\Delta \varphi_{a}$ and $+\Delta \varphi_{a}$ with the discrimination orientation. Of course, a combination of these two explanations could also be possible. For each subject and each condition we calculated the value of $\Delta \varphi_{\mathrm{s}}$ and $\Delta \varphi_{a}$ which corresponds with the bias measured in the present experiment (see Appendix A). We do not claim that subjects made a systematic orientation error or averaged the curvature over a certain area in order to judge the curvature along a specified orientation. We only determine how large the error or area would have been if they used one of these two methods. The predicted values of $\Delta \varphi_{\mathrm{s}}$ and $\Delta \varphi_{a}$ are given in Table 2. If subjects made a systematic orientation error, the error would have been on average $32^{\circ}$. If they averaged the curvature over a certain area, the orientation of the boundary lines would have been on average $-58^{\circ}$ and $+58^{\circ}$, which is more than one half of the surface area. It is hard to believe, though not impossible, that subjects made such large orientation errors. From a pilot experiment we know that subjects can feel the difference in the orientation of two identical cylinders quite well if the orientations of the surfaces differ by only $10^{\circ}$.

\section{Experiment 2}

In experiment 1 we found that the shape of a surface influenced the bias of curvature discrimination along a particular orientation. In this experiment we tested the

Table 2

The systematic orientation error $\Delta \varphi_{\mathrm{s}}$ and the angle $\Delta \varphi_{a}$ between the discrimination orientation and one of the boundary lines of the area over which subjects could have averaged the curvature, for both discrimination orientations. Because the area is assumed to be oriented symmetrically around the discrimination orientation, the total angle between the two boundary lines of the area is $2 \times \Delta \varphi_{a}$. The way in which the angles are calculated is described in Appendix A. When $\Delta \varphi_{a} \geqslant 90^{\circ}$ the curvature is averaged over the entire surface

\begin{tabular}{|c|c|c|c|c|}
\hline \multirow[t]{2}{*}{ Subject } & \multicolumn{2}{|c|}{$\Delta \varphi_{\mathrm{s}}$} & \multicolumn{2}{|l|}{$\Delta \varphi_{a}$} \\
\hline & $0^{\circ}$ & $90^{\circ}$ & $0^{\circ}$ & $90^{\circ}$ \\
\hline MK & $32^{\circ}$ & $36^{\circ}$ & $59^{\circ}$ & $66^{\circ}$ \\
\hline WR & $22^{\circ}$ & $8^{\circ}$ & $40^{\circ}$ & $13^{\circ}$ \\
\hline $\mathrm{RR}$ & $39^{\circ}$ & $13^{\circ}$ & $74^{\circ}$ & $22^{\circ}$ \\
\hline $\mathrm{BH}$ & $31^{\circ}$ & $39^{\circ}$ & $57^{\circ}$ & $74^{\circ}$ \\
\hline RN & $28^{\circ}$ & $40^{\circ}$ & $50^{\circ}$ & $76^{\circ}$ \\
\hline SW & $27^{\circ}$ & $54^{\circ}$ & $49^{\circ}$ & $>90^{\circ}$ \\
\hline average & \multicolumn{2}{|c|}{$32^{\circ}$} & \multicolumn{2}{|c|}{$58^{\circ}$} \\
\hline
\end{tabular}


validity of one of the possible explanations of the effect, namely that subjects made a systematic error of about $30^{\circ}$ in the discrimination orientation. Although we do not expect that subjects make such large orientation errors we want to be sure whether we can reject this hypothesis. Subjects were asked to discriminate the curvature of a doubly curved surface along the middle finger or across the middle finger, while the orientation of the surface was either $45^{\circ}$ or $-45^{\circ}$. Hence, it was not one of the principal curvatures that had to be discriminated. The curvature along the middle finger was the same for both surface orientations $\left(k\left(45^{\circ}\right)=k\left(-45^{\circ}\right)=\frac{1}{2} k_{\max }+\frac{1}{2} k_{\min }\right)$, only the principal curvatures $k_{\max }$ and $k_{\min }$ were interchanged (see Fig. 7). The curvature along the middle finger was also equal to the curvature across the middle finger $\left(k\left(45^{\circ}\right)=k\left(135^{\circ}\right)\right)$. If the orientation to which the subject attends deviates systematically, i.e. always towards the right or always towards the left of the discrimination orientation, the judgements will shift towards $k_{\max }$ for one orientation of the surface and towards $k_{\min }$ for the other orientation. Consequently, judgements will not be the same for the two orientations of the surface. If such a deviation exists, it would be

\section{discrimination orientation}

1) $0^{0}$

B) $45^{\circ}$
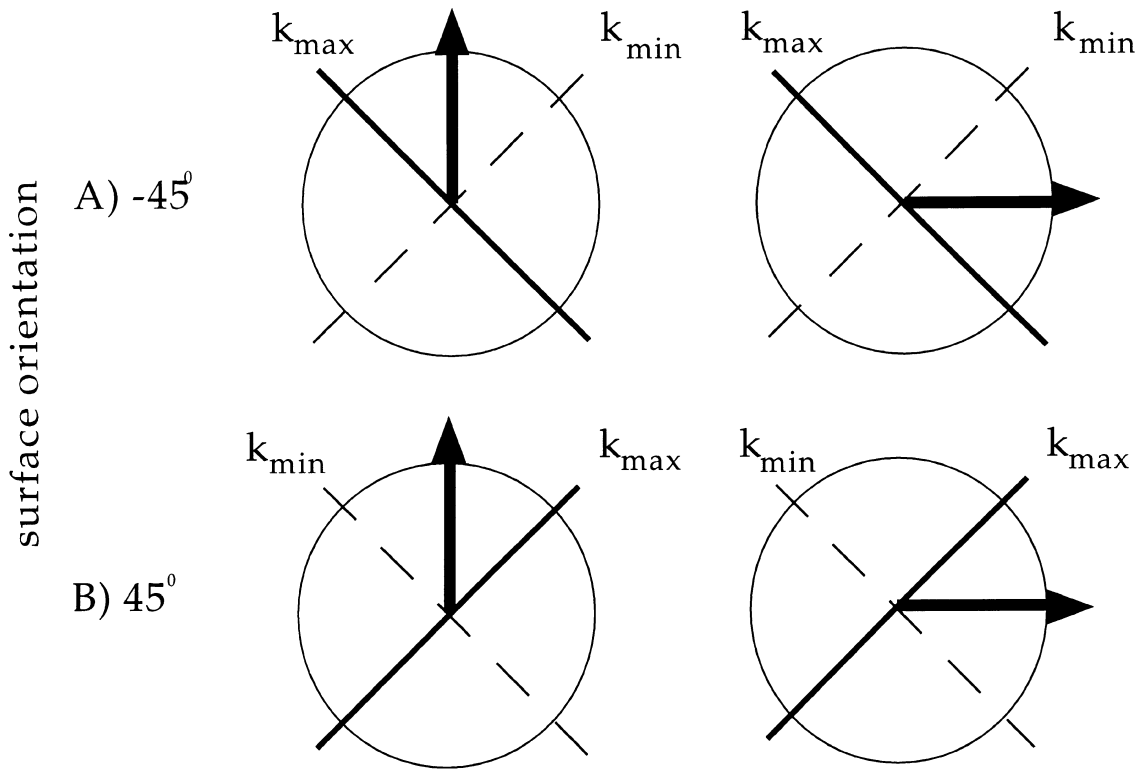

2) $90^{\circ}$

Fig. 7. The four conditions in which the doubly curved surfaces could be presented. The orientation of the surfaces could be $-45^{\circ}$ (a) or $45^{\circ}$ (b). The discrimination orientation could be $0^{\circ}$ (1) or $90^{\circ}$ (2). The curvatures in the discrimination orientations are the same for both orientations of the surface. The thick arrow points along the discrimination orientation, the solid line is the principal axis of $k_{\max }$, and the dashed line corresponds to the principal axis of $k_{\min }$. 
interesting to investigate whether the deviation depends on the asymmetry of the hand. In other words, if the orientation subjects actually pay attention to deviates towards the right for the right hand, does the orientation deviate then towards the left for the left hand? For each hand, the direction of the deviation could also depend on the discrimination orientation.

It is important to notice that if subjects average the curvature over a certain symmetrical area or if they do not make any orientation error, the results will be the same for both orientations of the surface.

\subsection{Method}

The stimuli and the experimental setup were identical to those in experiment 1 . Three subjects participated: MK, WR and RR. They also took part in the previous experiment. The doubly curved surfaces had an orientation of $-45^{\circ}$ or $45^{\circ}$ (see Fig. 7). The orientation was defined as the angle between the middle finger and the principal axis of $k_{\max }$. The sign of the angle is negative (positive) when $k_{\max }$ is on the left (right) of the middle finger. Subjects had to attend to the orientation of the middle finger and to compare the curvature of a doubly curved surface along that orientation with that of a flat surface. In order to investigate whether the orientation error was always in the same direction, one subject performed the same experiment with his left hand, and, in another session he had to attend to the orientation across the middle finger of his right hand. Unlike the previous experiment, surfaces with different surface orientations are not treated as different stimuli but as different conditions, because the curvatures to be discriminated are the same. All stimuli were presented 10 times in each condition. The experiment involved about $16 \mathrm{~h}$ in total.

\subsection{Results}

In Fig. 8 the results of an ideal observer giving veridical answers are shown. Again the percentages of judgements in which the doubly curved surfaces were judged to be more convex than the flat one along the middle finger (or across the middle finger) are represented by gray values. Since $k_{\max } \geqslant k_{\min }$, only one half of the diagram is needed to represent all surfaces. Therefore, the data for the $-45^{\circ}$ orientation are plotted in the upper-left corner of the figure, and the data for the $45^{\circ}$ orientation are presented in the lower-right corner. Both the horizontal and the vertical axes correspond to the principal curvature of either $k_{\max }$ or $k_{\min }$, depending on the orientation of the surface. For both surface orientations the curvature to be discriminated is $k_{\mathrm{d}}=\frac{1}{2} k_{\max }+\frac{1}{2} k_{\min }$. Therefore, the percentage of judgements "more convex" is 100 , 50 or 0 when $k_{\max }+k_{\min }>0, k_{\max }+k_{\min }=0$, and $k_{\max }+k_{\min }<0$, respectively. Because for an ideal observer there is no effect of orientation, the plot is symmetric with respect to the line $k_{\max }=k_{\min }$.

In Fig. 9 the judgements of subject RR are presented for the $-45^{\circ}$ and $45^{\circ}$ conditions. The plot seems to be somewhat asymmetric. For the other two subjects the 


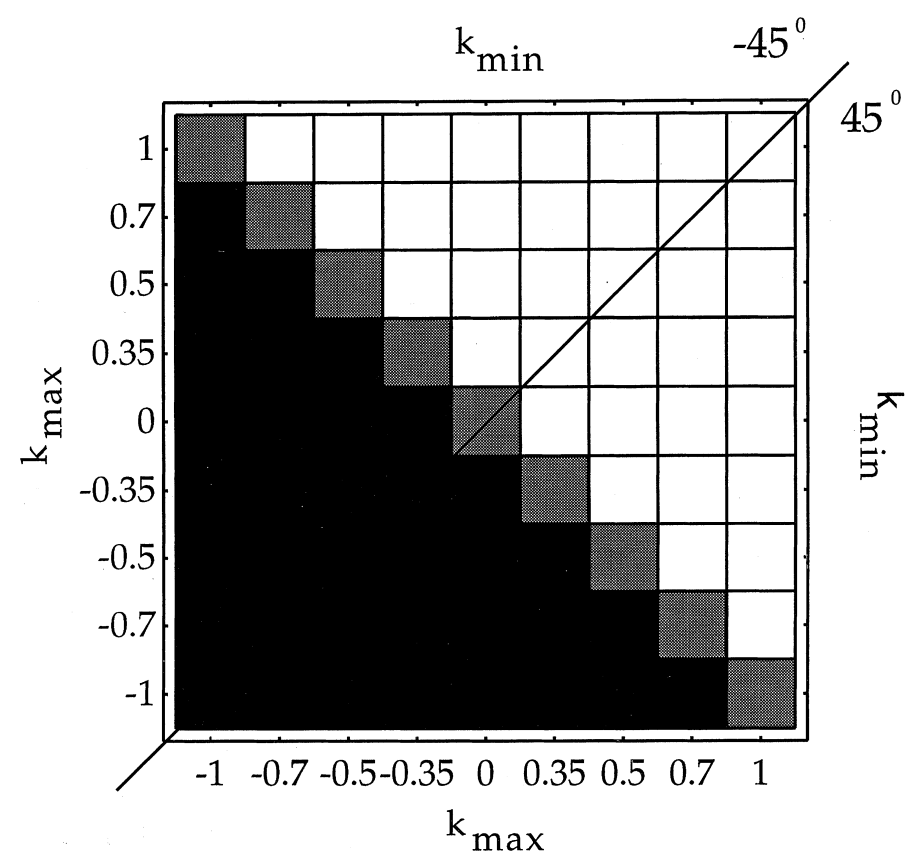

Fig. 8. The percentage of judgements in which an ideal observer would judge the doubly curved surfaces to be more convex than the flat reference surface along the middle finger or across the middle finger. Surfaces in the upper-left corner had an orientation of $-45^{\circ}$, surfaces in the lower-right corner had an orientation of $45^{\circ}$. Since $k_{\max } \geqslant k_{\min }$, the horizontal and vertical axes are not the same for the two orientations. For the oblique line which divides the two orientations it holds that $k_{\max }=k_{\min }$.

asymmetry was more apparent. In order to test whether the plots were significantly asymmetric, i.e. whether the judgements for the two orientations were significantly different, we performed a paired $t$-test. In other words, for each surface the percentage of judgements "more convex" in the $-45^{\circ}$ condition was compared with the percentage in the $45^{\circ}$ condition. Then it was tested whether the difference between the two orientations averaged over the surfaces was significantly different from zero. The spherical surfaces were not included in the analysis, since for these surfaces the orientation is not defined. Hence we had a total of 32 surfaces. Table 3 shows the average difference between the two surface orientations and the $t$-value, for each subject. If the difference is close to $0 \%$, the curvature along the middle finger of a surface with an orientation of $-45^{\circ}$ was discriminated in the same way as a surface had an orientation of $45^{\circ}$. The maximum difference can be $100 \%$. The difference between the two orientations of the surface was significant for all subjects $(t(31)>2.75, p<0.01)$.

Since subjects judged the curvature along the middle finger differently for a surface with an orientation of $45^{\circ}$ and $-45^{\circ}$, they could have made a systematic error $\left(\Delta \varphi_{\mathrm{s}}\right)$ in the discrimination orientation. In order to calculate the error in the discrimination orientation we fitted a psychometric function to the raw data (Eq. 


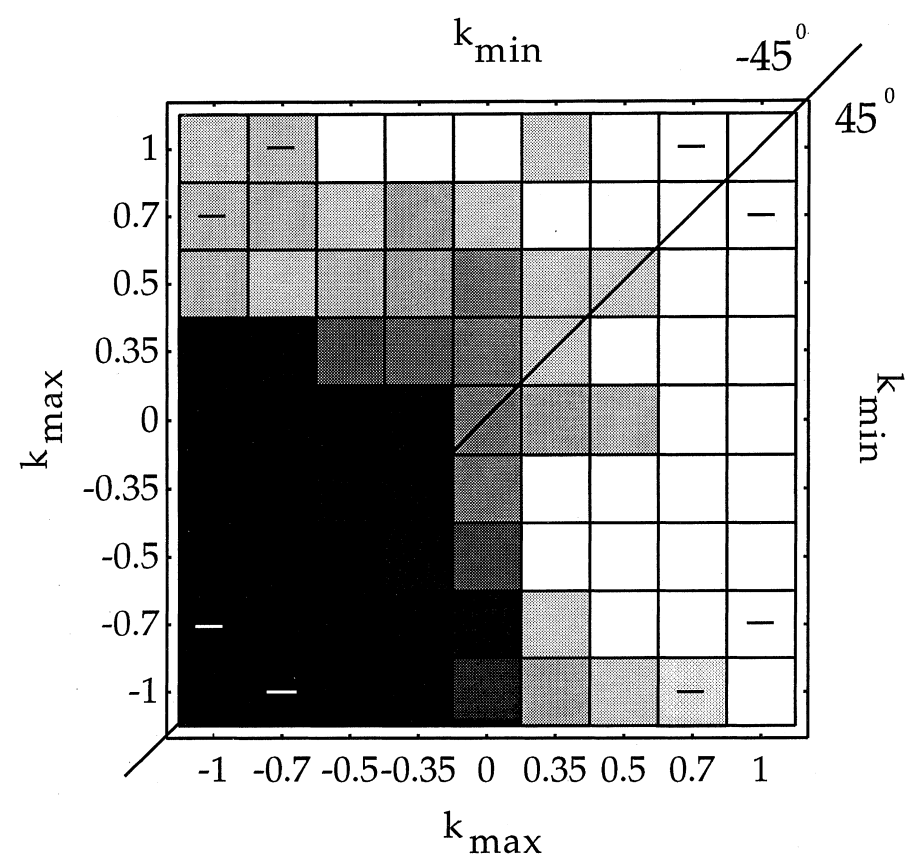

Fig. 9. The percentage of judgements "more convex" for subject RR in the case where the orientation of the surfaces was $-45^{\circ}$ or $45^{\circ}$. The subject had to attend to the orientation of the middle finger. The squares with a minus sign represent surfaces that were not presented. In order to prevent these 'empty' squares blurring a possible trend, we gave these squares a gray value equal to the average of the three neighbouring squares.

\section{Table 3}

Average differences in the percentage of judgements for doubly curved surfaces with an orientation of $-45^{\circ}$ and $45^{\circ}$, and $t$-values obtained from a paired $t$-test $(d f=31)$. The difference is positive when the percentage of judgements "more convex" is larger for the $45^{\circ}$ orientation. Subjects had to attend to the orientation of the middle finger $\left(0^{\circ}\right)$, or perpendicular to the middle finger $\left(90^{\circ}\right)$. One subject also touched the surfaces with his left hand for the $0^{\circ}$ condition

\begin{tabular}{lcc}
\hline Subject & Difference $(\%)$ & $t(31)$ \\
\hline MK $\left(0^{\circ}\right)$ & 32 & 5.2 \\
MK $\left(90^{\circ}\right)$ & 17 & 4.6 \\
MK $\left(0^{\circ} /\right.$ eft $)$ & -38 & 4.6 \\
WR $\left(0^{\circ}\right)$ & 31 & 5.1 \\
RR $\left(0^{\circ}\right)$ & 18 & 5.7 \\
\hline
\end{tabular}

3). We substituted $k_{\mathrm{d}}$ by $k_{\max } \cos ^{2}\left(\varphi_{\mathrm{s}}\right)+k_{\min } \sin ^{2}\left(\varphi_{\mathrm{s}}\right)$, with $\varphi_{\mathrm{s}}=45^{\circ}+\Delta \varphi_{\mathrm{s}} \cdot{ }^{5} \mathrm{In}$ Table 4 the fitted parameters $\Delta \varphi_{\mathrm{s}}, \mu$ and $\sigma$ are given for each subject. If $\Delta \varphi_{\mathrm{s}}$ is

\footnotetext{
${ }^{5}$ We also fitted a more general function in which $k_{\mathrm{d}}$ was substituted by $a k_{\max }+b k_{\min }$. The values of $\Delta \varphi_{\mathrm{s}}$ calculated via the parameters $a$ and $b$ were almost the same.
} 
Table 4

Values of the parameters fitted to the data: The error in the discrimination orientation $\left(\Delta \varphi_{\mathrm{s}}\right)$, the bias $(\mu)$ and the threshold of curvature discrimination $(\sigma)$

\begin{tabular}{lccc}
\hline Subject & $\Delta \varphi_{\mathrm{s}}$ & $\mu\left(\mathrm{m}^{-1}\right)$ & $\sigma\left(\mathrm{m}^{-1}\right)$ \\
\hline MK $\left(0^{\circ}\right)$ & $12^{\circ}$ & 0.03 & 0.20 \\
MK $\left(90^{\circ}\right)$ & $-6^{\circ}$ & 0.05 & 0.22 \\
MK $\left(0^{\circ} / \mathrm{left}\right)$ & $-20^{\circ}$ & 0.15 & 0.21 \\
WR $\left(0^{\circ}\right.$ & $15^{\circ}$ & 0.01 & 0.22 \\
RR $\left(0^{\circ}\right)$ & $5^{\circ}$ & -0.19 & 0.19 \\
\hline
\end{tabular}

positive, the discrimination orientation is rotated clockwise. The error in the discrimination orientation is on average $12^{\circ}$. The average discrimination threshold is $0.21 \mathrm{~m}^{-1}$.

\subsection{Discussion}

When subjects touched a doubly curved surface such that the principal axis of $k_{\max }$ was $45^{\circ}$ on the left of their middle finger they judged the curvature along the middle finger (or across the middle finger) differently than when $k_{\max }$ was $45^{\circ}$ on the right of their middle finger, whereas the objective curvatures were the same. This suggests that subjects made an error in the orientation along which they had to discriminate curvature. The error ranged between $5^{\circ}$ and $15^{\circ}$ clockwise when the discrimination orientation corresponded to the orientation of the right middle finger. In order to test whether the deviation was always clockwise, irrespective of the discrimination orientation and the hand that touched the surface, one subject was instructed to attend to the orientation across the right middle finger or to the orientation along the left middle finger, in two separate experiments. The deviation was anticlockwise in both cases. Because the orientation error seems to depend on the discrimination orientation, it cannot be due to a constant rotation of haptic orientation. Since the deviation was symmetrical for the two hands, it could be a mechanical or a sensory effect.

Of course we do not know with certainty that subjects attended to an orientation other than the designated one, although the data can be described well by this assumption. What we do know is that if they made an error in the discrimination orientation, the error would have been about $10^{\circ}$. The influence of shape on curvature perception along a particular orientation, demonstrated in the previous experiment, could be explained by an orientation error of about $30^{\circ}$. Since this error is larger by a factor three, it is very unlikely that the influence of shape is due only to an orientation error. So, although subjects might have attended to a slightly different orientation, other factors probably contribute to the effect as well.

Thresholds for curvature discrimination were similar to those of the $0^{\circ}$ condition in experiment $1\left(0.20 \mathrm{~m}^{-1}\right)$. Since the only difference between the two experiments was the orientation of the surface $\left(0^{\circ}\right.$ or $90^{\circ}$ and $-45^{\circ}$ or $\left.45^{\circ}\right)$, discrimination thresholds are apparently independent of the orientation of the surface for a certain 
fixed discrimination orientation. This means that it is not easier to discriminate the curvature along a principal axis.

\section{General discussion}

In this paper we demonstrated that thresholds of curvature discrimination along a particular orientation of a doubly curved surface depend on the effective contact length, i.e. the length of the contact area with the surface along that orientation, but not on the shape of the surface. However, the bias of curvature discrimination was shown to depend strongly on the shape of the surface. Subjects judged a principal curvature to be more convex when the perpendicular curvature was convex than when this curvature was concave. We found that this effect was not due only to a systematic error made by the subject regarding the discrimination orientation.

Hunter (1954) and Davidson (1972) already reported that dynamic curvature perception is biased. They found that the way in which subjects explored a curved edge influenced the veridicality and accuracy of the judgements. Generally, the edge was judged to be straight when it was curved away from the subject. Kappers et al. (1994) observed that dynamic shape identification was not always veridical. Although they did not measure the bias systematically, they noticed that the bias varied from subject to subject and from shape to shape. Vogels et al. (1996) demonstrated that the statically judged curvature of a spherical surface was strongly influenced by a previously touched surface. Subjects more often judged a flat surface to be convex after they had touched a concave surface than after touching a convex surface.

We wondered whether the influence of shape on the perceived curvature along a particular orientation has consequences for shape perception. If subjects determine the shape of a doubly curved surface by comparing the principal curvatures ${ }^{6}$ the perceived shape would not be veridical because we found that the principal curvatures are not perceived veridically. Since the perceived curvatures along the principal axes are biased towards each other, the difference between the two curvatures would be perceived to be smaller and the shape would be perceived to be more symmetrical than it actually is. In an experiment reported in Vogels (1997) we investigated whether shape perception was biased in the direction expected from the results of the present study. Subjects were instructed to judge by means of static touch which of two successively presented surfaces (a doubly curved surface and a flat one) was more symmetrical. Since we used the same subjects as in this paper we could calculate the expected bias. The results showed that shape perception was biased but

\footnotetext{
${ }^{6}$ It is reasonable to assume that subjects actually use this strategy because Kappers et al. (1994) reported that when subjects had to identify the shape of a surface actively, they usually rotated the shape such that one of the principal axes became aligned with the fingers and then compared the principal curvatures. Also in the case of static touch, Pont et al. (1998b) found indications that subjects compare principal curvatures in order to judge shape.
} 
mostly not in the predicted direction. The shape perception experiment combined with the results of the present study suggests that perceived shape is not determined by simply comparing the perceived curvatures in the principal directions. That is, the perceived shape does not depend on the perceived principal curvatures in the same way as shape mathematically depends on the principal curvatures. However, there can still be an (unknown) relation between perceived shape and perceived principal curvatures.

Pont et al. (1998b) concluded that similar mechanisms underlie the perception of curvature and shape. They found that shape perception could be described in terms of the difference in the growth of curvature perception with increasing curvature along and across the fingers and the difference in the phenomenal flatness (of a cylinder) along these orientientions. We showed that the phenomenal flatness along a particular orientation is not constant but depends on the shape of the surface. Since the bias of shape perception could not be described in terms of the influence of shape on the perceived curvature, the mechanisms of curvature and shape perception are not similar in every respect.

So far, we have no explanation for the influence of shape on curvature perception. Since discrimination thresholds were much smaller for doubly curved surfaces than for curved strips (Pont et al., 1997), we concluded that subjects combined information from different parts of the hand in order to discriminate the curvature along a particular orientation. It could be that the information from each part of the hand is affected by the shape, or that only the combined information is disturbed. Therefore, it would be interesting to present subjects with stimuli that consist of two perpendicular strips that are curved. In such a situation, subjects cannot make an error in the discrimination orientation, nor can they average the curvature over a certain area. If the haptic system is not able to restrict processing to a single region of the hand, the curvature to be discriminated would still be influenced by the perpendicular curvature. Another idea is to investigate whether the effect depends on the size of the contact area. If the effect does not arise when subjects touch doubly curved surfaces with one fingertip, ${ }^{7}$ it is likely that the combining of information from relatively large areas is a determining factor. Clearly, future research is needed to understand the underlying mechanisms better.

\section{Acknowledgements}

The authors are grateful to the Netherlands Organization of Scientific Research (NWO) for funding this project. This research was conducted while I.M.L.C. Vogels was supported by a grant from the Foundation for Behavioural and Educational Sciences of this organization (575-62-054), awarded to Dr A.M.L. Kappers.

\footnotetext{
${ }^{7}$ Since the threshold of curvature discrimination with the fingertip is about $5 \mathrm{~m}^{-1}$ (Goodwin et al., 1991) the surfaces used in our experiments are not suitable for such an experiment.
} 


\section{Appendix A}

The error $\Delta \varphi_{\mathrm{s}}$ in the discrimination orientation can be calculated in the following way: Since the bias of curvature discrimination is $\mu_{\mathrm{d}}=a_{0}+a_{1} k_{\mathrm{p}}$, with $a_{0}$ not significantly different from zero, the curvature to be discriminated is perceived as flat when

$$
k_{\mathrm{d}}=a_{1} k_{\mathrm{p}}
$$

If subjects make an error of $\Delta \varphi_{\mathrm{s}}$, the curvature along the orientation to which they really pay attention is flat when

$$
k\left(\Delta \varphi_{\mathrm{s}}\right)=k_{\mathrm{d}} \cos ^{2}\left(\Delta \varphi_{\mathrm{s}}\right)+k_{\mathrm{p}} \sin ^{2}\left(\Delta \varphi_{\mathrm{s}}\right)=0 .
$$

If we substitute Eq. A.1 into Eq. A.2 we get

$$
\frac{\sin ^{2}\left(\Delta \varphi_{\mathrm{s}}\right)}{\cos ^{2}\left(\Delta \varphi_{\mathrm{s}}\right)}=-\frac{k_{\mathrm{d}}}{k_{\mathrm{p}}}=-a_{1} .
$$

Hence

$$
\Delta \varphi_{\mathrm{s}}=\arctan \left(\sqrt{-} a_{1}\right) .
$$

The angle $\Delta \varphi_{a}$ was computed as follows: if subjects average the curvature over an area of which the boundary lines make an angle of $-\Delta \varphi_{a}$ and $+\Delta \varphi_{a}$ with the discrimination orientation, the averaged curvature will be

$$
k_{\mathrm{av}}=\frac{\int_{-\Delta \varphi_{a}}^{\Delta \varphi_{a}} k(\varphi) \mathrm{d} \varphi}{\int_{-\Delta \varphi_{a}}^{\Delta \varphi_{a}} \mathrm{~d} \varphi}
$$

with $k(\varphi)=k_{\mathrm{d}} \cos ^{2}(\varphi)+k_{\mathrm{p}} \sin ^{2}(\varphi)$. If we compute the integral, substitute Eq. A.1 and set $k_{\mathrm{av}}$ to zero we get

$$
\Delta \varphi_{a}=\frac{1}{2} \sin \mathrm{c}^{-1}\left(\frac{1+a_{1}}{1-a_{1}}\right) .
$$

It can be shown that approximately $\Delta \varphi_{a}=\sqrt{3} \Delta \varphi_{\mathrm{s}}$.

\section{References}

Coren, S., 1993. The Left-Hander Syndrome. Vintage Books, New York.

Davidson, P.W., 1972. Haptic judgements of curvature by blind and sighted humans. Journal of Experimental Psychology 93, 43-55.

Goodnow, J.J., Baum, B., Davidson, P., 1971. A haptic error: Skew in a symmetrical curve. Perception and Psychophysics 10, 253-256.

Goodwin, A.W., John, K.T., Marceglia, A.H., 1991. Tactile discrimination of curvature by humans using only cutaneous information of the fingerpads. Experimental Brain Research 86, 663-672.

Gordon, I.A., Morison, V., 1982. The haptic perception of curvature. Perception and Psychophysics 31, 446-450.

Hunter, I.M.L., 1954. Tactile-kinaesthetic perception of straightness in blind and sighted humans. Quarterly Journal of Experimental Psychology 6, 149-154. 
Kappers, A.M.L., Koenderink, J.J., 1996. Haptic unilateral and bilateral discrimination of curved surfaces. Perception 25, 739-749.

Kappers, A.M.L., Koenderink, J.J., Lichtenegger, I., 1994. Haptic identification of curved surfaces. Perception and Psychophysics 56, 53-61.

LaMotte, R.H., Srinivasan, M.A., 1993. Responses of cutaneous mechanoreceptors to the shape of objects applied to the primate fingerpad. Acta Psychologica 84, 41-51.

LaMotte, R.H., Srinivasan, M.A., Lu, C., Klusch-Petersen, A., 1994. Cutaneous neural codes for shape. Canadian Journal of Physiology and Pharmacology 72, 498-510.

Lederman, S.J., Klatzky, R.L., 1987. Hand movements: A window into haptic object recognition. Cognitive Psychology 19, 342-368.

Matthews, P.B.C., 1988. Proprioceptors and their contribution to somatosensory mapping: Complex messages require complex processing. Canadian Journal of Physiology and Pharmacology 66, 430-438.

Pont, S.C., Kappers, A.M.L., Koenderink, J.J., 1998a. Similar mechanisms underlie curvature comparison by static and dynamic touch. Accepted for Perception and Psychophysics.

Pont, S.C., Kappers, A.M.L., Koenderink, J.J., 1998b. Anisotropy in haptic curvature perception. Accepted for Perception.

Pont, S.C., Kappers, A.M.L., Koenderink, J.J., 1997. Haptic curvature discrimination of several regions of the hand. Perception \& Psychophysics 8, 1225-1240.

Press, W.H., Flannery, B.P., Teukolsky, S.A., Vetterling, W.T., 1988. Numerical Recipes in C. Cambridge University Press, Cambridge, MA.

Srinivasan, M.A., LaMotte, R.H., 1987. Tactile discrimination of shape: Responses of slowly and rapidly adapting mechanoreceptive afferents to a step indented into the monkey fingerpad. Journal of Neuroscience 7, 1682-1697.

Vogels, I.M.L.C., 1997. Haptic After-effect of Curved Surfaces. Thesis Universiteit Utrecht, Helmholtz Instituut, pp. 82-89.

Vogels, I.M.L.C., Kappers, A.M.L., Koenderink, J.J., 1996. Haptic after-effect of curved surfaces. Perception 25, 109-119. 